\title{
Quantum trajectory analysis of a thresholdlike transition in the microlaser
}

\author{
Changhuei Yang and Kyungwon An \\ George R. Harrison Laboratory, Massachusetts Institute of Technology, Cambridge, Massachusetts 02139
}

(Received 29 July 1996)

\begin{abstract}
In a recent microlaser experiment [K. An et al., Phys. Rev. Lett. 73, 3375 (1994)], a thresholdlike transition of intracavity mean photon number as a function of intracavity mean atom number has been observed. In this paper the behavior is explored with quantum trajectory simulations. It is shown that the transition is caused by enhanced atom-cavity Rabi interaction due to the increase of the intracavity photon number as the intracavity atom number is increased. The transition is further accentuated by the position-dependent variation of the coupling constant in the Fabry-Pérot cavity. In addition, it is demonstrated that multiatom collective effects are negligible in the microlaser under consideration, in which atoms are injected into the cavity at random times and the product of the coupling constant and atom-cavity interaction time is much less than $\pi$. In this case the analytic theory of the one-atom micromaser [P. Filipowicz et al., Phys. Rev. A 34, 3077 (1986)] can be extrapolated into the multiatom region, assuming uniform atom-cavity coupling throughout the cavity and monovelocity atomic injection. Finally, simulations are performed which account for spatial variation of coupling constant, velocity distribution of injected atoms, and spontaneous atomic decay in the actual experiment. The results are in good agreement with experiment. [S1050-2947(97)03506-3]

PACS number(s): 42.50.-p, 42.55.-f, 32.80.-t
\end{abstract}

\section{INTRODUCTION}

Cavity quantum electrodynamics (QED) [1] has been a subject of extensive experimental and theoretical studies in recent years. In this field the interaction of an atom with a single mode cavity is particularly important. An atom in a cavity constitutes the simplest form of light-matter interaction, and often serves as an ideal testing ground for fundamental studies. It is an attractive setup for studying atomphoton entanglement, Schrödinger's cat states [2], and quantum computing [3].

The microlaser [4] is an interesting setup for studying cavity QED with a single atom in the few quantum limit. The photon generation process in the microlaser is the coherent atom-cavity Rabi interaction described by the JaynesCummings model [5]; the process is significantly different from that of a conventional laser characterized by spontaneous and stimulated emission, and therefore the microlaser has the potential to exhibit many features of quantum optics, such as photon-number trapped states, squeezing, and atomfield entanglement. In addition, the microlaser provides new experimental opportunities not available in its microwave counterpart, the micromaser [6]: photon detection capability in the microlaser makes possible the direct measurement of intracavity mean photon number, emission spectrum [7], higher-order correlation functions of the output emission, and atom-field correlation function.

Extending the single-atom microlaser to the many-atom situation is also an interesting problem. With increasing number of atoms, novel mesoscopic quantum mechanical features can arise. Although multiatom operation can be catastrophic to achieving photon-number trapped states [8], enhanced atom-cavity interaction due to collective interaction of atoms with the common cavity field can lead to highly nonclassical photon statistics [9].

Thresholdless lasing is one novel feature one expects from such mesoscopic laser devices. Researchers have been trying to realize thresholdless lasing in semiconductor microcavities for many years $[10,11]$. Because of the intrinsic complexity of such condensed matter devices, it is rather difficult to obtain a good understanding of the underlying physics. Hence one strategy is to first understand a more fundamental and simpler system, and then apply the acquired knowledge to more complex systems. The microlaser can serve as a testing ground for such ideas, since it can be easily extended to many-atom operation but is still simple enough to allow fairly rigorous theoretical study.

In the microlaser experiment, a stream of barium atoms $\left({ }^{138} \mathrm{Ba}\right)$ is injected into a high- $Q$ optical resonator. Prior to entry, each atom is excited by a $\pi$-pulse laser field into a long-lived excited state $\left(6{ }^{1} S_{0} \rightarrow 6{ }^{3} P_{1}\right)$. While in the cavity, the atoms couple to the cavity mode and undergo coherent atom-cavity interaction. As each atom leaves, there is a certain probability (hereafter called the emission probability $\left.A_{\text {em }}\right)$ that a photon is left behind in the cavity. Note that $A_{\text {em }}$ is a result of coherent atom-cavity interaction characterized by the atom-cavity coupling constant $g$; the contribution to $A_{\text {em }}$ due to usual spontaneous emission (in the absence of atom-cavity coupling) is extremely small $\left(\sim 10^{-6}\right)$. As will be seen below, $A_{\mathrm{em}}$ is an important parameter in understanding the microlaser operation.

If there exist exactly $n$ photons in the microlaser, the Rabi-oscillation frequency becomes $2 \sqrt{n+1} g$. In the currently realized microlaser experiment, atoms optimally coupled to the cavity undergo approximately $1 / 6$ of a complete Rabi cycle when the cavity is initially empty. Once a photon is emitted into the cavity, the next atom will experience a Rabi frequency enhanced by the $\sqrt{n+1}$ factor, and hence have an increased emission probability. This can lead to a rapid increase of intracavity mean photon number $\langle n\rangle$ at $\langle n\rangle \sim 1$, when $\langle n\rangle$ is plotted as a function of intracavity mean atom number $\langle N\rangle$. Such a trend resembles the threshold transition of a conventional laser in that output suddenly increases as the pumping rate exceeds a threshold. However, 


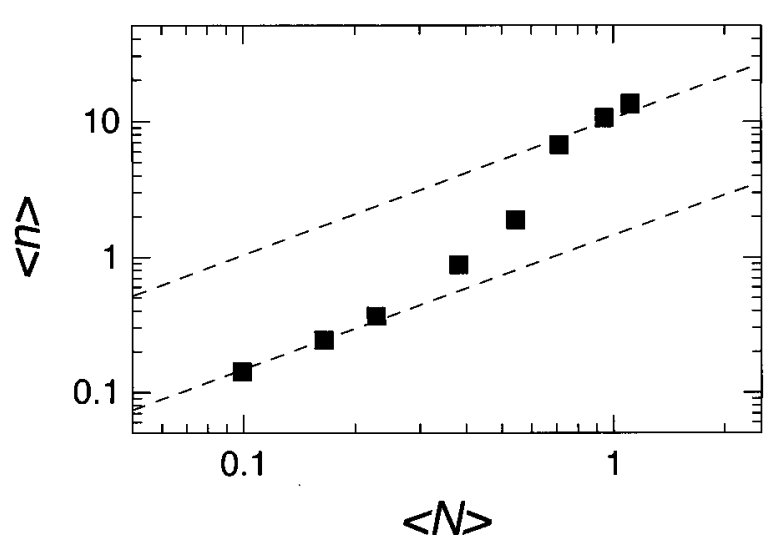

FIG. 1. Experimental results from Ref. [4] Parameters: $2 \kappa=2 \pi \times 190 \mathrm{kHz} ; g_{0}=2 \pi \times 360 \mathrm{kHz}$; mean velocity of thermal atoms, $365 \mathrm{~m} / \mathrm{s}$; mode waist, $43 \mu \mathrm{m}$.

we wish to emphasize that the thresholdlike transition in the microlaser is entirely different in origin, and is far less pronounced than ordinary threshold transitions. Such mild thresholdlike transitions have been observed when the microlaser operates with a few atoms, as shown in Fig. 1, excerpted from Ref. [4].

There have been speculations that the thresholdlike transition observed in the microlaser experiment might be due to many-atom collective effects. Such speculation first appeared in Ref. [4] as an explanation for the discrepancy between theory and experiment. In this work, however, we show that the microlaser thresholdlike transition is not due to the many-atom collective effects but due to the enhanced Rabi oscillations (with $\sqrt{n+1}$ factor), and is further accentuated by the position dependence of $g$ in the cavity. We demonstrate that many-atom collective effects, in fact, do not play any significant role in the microlaser experiment under consideration. A stochastic wave function method is used as an analytical tool in the present work.

The newly developed quantum trajectory simulation (QTS) formalism provides a practical simulation tool for analyzing a system which has dissipative processes but possesses only a few degrees of freedom $[12,13]$. Hence it is particularly suitable for analyzing the microlaser, which consists of one or a few atoms coupled to a single cavity mode. In QTS, the atom-cavity Schrödinger equation is numerically integrated for a long succession of injected atoms. Dissipative processes - atomic and cavity decay - are simulated through a stochastic wave function method. There have been numerous works with QTS; they include studies on the Stark splitting of the Rabi resonance for an atom strongly coupled to a cavity [14], studies of spontaneous emission of atoms under excitation from laser light [15], and modeling of the fluorescence spectrum of atoms in a magneto-optic trap [16].

In Sec. II we extend the QTS formalism in order to describe a microlaser operating with many atoms. The hallmark of this extended formalism is that the atom-field entangled wave function is continuously adjusted to account for atomnumber fluctuations in the cavity, due to the fact that atoms are continuously injected and removed.

To begin the analysis, we first apply the micromaser theory [17] to an idealized microlaser model in Sec. III A, and we then study the mechanism causing the thresholdlike transition, namely, the enhanced Rabi-oscillation process. The micromaser theory involves solving the Schrödinger equation with the Jaynes-Cummings Hamiltonian when the atom is in the cavity and letting the cavity field decay exponentially when no atom is present. The atom-field coupling is assumed to be uniform throughout the cavity in this theory.

In the actual experiment, the coupling constant has nonuniform spatial profile: standing-wave variations along the cavity axis and a Gaussian mode profile in the transverse directions. In Sec. III B we show that the nonuniform coupling constant enhances the thresholdlike transition. This enhancement of the transition in a standing-wave cavity was first pointed out elsewhere [18], approximating the standingwave mode by assuming that half of the atoms are at the nodes and half at the antinodes. It was shown that as $\langle n\rangle$ builds up, the coupling enhancement due to more photons in the cavity prompts even weakly coupled atoms to participate in laser emission. The result would then be a more distinct thresholdlike transition. The same reasoning holds for the Gaussian profile in the transverse directions.

In Sec. III C we examine many-atom effects and show that those effects are negligible for the microlaser under consideration, and hence that the results of one-atom micromaser theory can be extrapolated to the many-atom region under proper conditions. In Sec. III D the microlaser experiment is analyzed with fully extended simulations including cavity mode structure, atomic decay, and velocity distribution. This analysis supersedes the previous one $[4,18]$, serving as a recalibration method of the photon and atom detection system used in the experiment.

\section{EXTENDED QUANTUM TRAJECTORY FORMALISM}

Consider a microlaser operating with many atoms, for which the Hamiltonian can be written as

$$
\begin{gathered}
H=H_{\mathrm{JC}}+H_{D}, \\
H_{\mathrm{JC}}=i \hbar \sum_{q=1}^{N_{0}} g\left(\overrightarrow{r_{q}}\right)\left(\sigma_{q-} a^{\dagger}-\sigma_{q+} a\right), \\
H_{D}=-i \hbar \frac{\kappa}{2} a^{\dagger} a-i \hbar \sum_{q=1}^{N_{0}}\left(\gamma \sigma_{q+} \sigma_{q-}+\gamma^{\prime} \sigma_{q+}^{\prime} \sigma_{q-}^{\prime}\right),
\end{gathered}
$$

where $g\left(\overrightarrow{r_{q}}\right)$ is the position-dependent coupling constant of the $q$ th atom at position $\vec{r}_{q}, \kappa$ is the cavity decay rate, $2 \gamma$ is the atomic decay rate from $6{ }^{3} P_{1}$ to the ground state $6{ }^{1} S_{0}$ and $2 \gamma^{\prime}$ is the atomic decay rate from $6{ }^{3} P_{1}$ to $5{ }^{3} D_{2,1}$ states. (See Fig. 2 for the energy level diagram of atomic barium.) $\sigma_{q \pm}$ are the raising and lowering operators for the transition between $6{ }^{3} P_{1}$ and $6{ }^{1} S_{0}$, while $\sigma_{q \pm}^{\prime}$ are the raising and lowering operators for the transition between $6{ }^{3} P_{1}$ and $5{ }^{3} D_{2,1} . a^{\dagger}$ and $a$ are the creation and annihilation operators of the photons in the cavity. $N_{0}$ is the number of atoms in a volume $V_{0}$, which is large enough to completely enclose the cavity mode so that the atoms outside this volume have negligibly weak atom-cavity coupling constants. The Hamiltonian $H_{\mathrm{JC}}$ accounts for the atom-cavity interaction whereas $H_{D}$ is a Hamiltonian associated with atom and cavity decay. It is important to note that as atoms drift into 


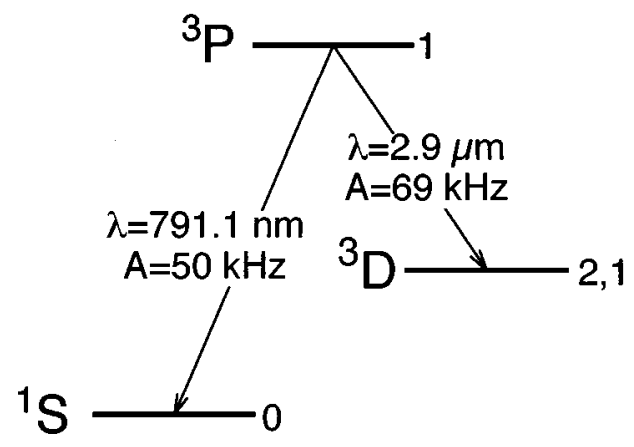

FIG. 2. Energy-level diagram of atomic barium.

and out of the volume $V_{0}$, new terms are constantly added and old terms are constantly removed from $H_{\mathrm{JC}}$ and $H_{D}$.

Coherent evolution of the wave function $|\Psi(t)\rangle$ due to $H_{\mathrm{JC}}$ is obtained by numerically integrating the Schrödinger equation. If the time interval of integration is small enough, the wave function at time $t+\Delta t$ is related to the wave function at time $t$ by

$$
\begin{aligned}
|\Psi(t+\Delta t)\rangle \simeq \mid & |\Psi(t)\rangle+\sum_{q=1}^{N_{0}} g\left(\overrightarrow{r_{q}}\right) \\
& \times\left(\sigma_{q-} a^{\dagger}-\sigma_{q+} a\right) \Delta t|\Psi(t)\rangle .
\end{aligned}
$$

The treatment of the dissipative Hamiltonian $H_{D}$ follows that of Ref. [14]. Specifically, the probability of cavity decay in each time interval is computed by multiplying the decay rate of a photon out of the cavity with the time interval $\Delta t$ :

$$
r_{c}(t) \Delta t=\kappa\left\langle\Psi(t)\left|a^{\dagger} a\right| \Psi(t)\right\rangle \Delta t
$$

where $\left\langle\Psi(t)\left|a^{\dagger} a\right| \Psi(t)\right\rangle$ is the cavity photon expectation number and will henceforth be denoted as $\langle n\rangle$. The probability of cavity decay is then compared with a random number between 0 and 1 . If the random number is less than this probability, it is determined that a photon has decayed out of the cavity. We then modify the wave function by applying the annihilation operator $a$ to it and the resulting wave function is renormalized:

$$
|\Psi(t)\rangle \rightarrow a|\Psi(t)\rangle .
$$

Otherwise, the wave function is modified as follows and then renormalized:

$$
|\Psi(t)\rangle \rightarrow\left(1-\frac{\kappa}{2} \Delta t a^{\dagger} a\right)|\Psi(t)\rangle .
$$

This null observation adjustment can be understood as a reduction of the probability of higher photon-number state due to the additional information that no photon is observed during the time interval. The incorporation of atomic decay follows a similar procedure, where we replace the photon creation and annihilation operators with atomic raising and lowering operators, respectively, and the cavity decay rate with the atomic decay rates.

In this numerical simulation scheme, the entry of an atom into the cavity requires two actions. The first is addition of the respective atom-cavity interaction term to the Hamiltonian $H$. The second is the concatenation of the wave func- tion of the injected atom into the system wave function. The wave function of an $N_{0}$ atom-cavity system can be expressed as

$$
\begin{aligned}
\left|\Psi_{N_{0}}(t)\right\rangle= & \sum_{n=0}^{\infty} \underbrace{\sum_{\phi_{1}=0}^{1} \ldots \sum_{\phi_{N_{0}}=0}^{1}}_{\text {sum over } N_{0} \text { atoms }} A_{n, \phi_{1}, \phi_{2} \ldots \ldots \phi_{N_{0}}}(t) \\
& \times|n\rangle\left|\phi_{1}\right\rangle\left|\phi_{2}\right\rangle \cdots\left|\phi_{N_{0}}\right\rangle,
\end{aligned}
$$

where $|n\rangle$ denotes the cavity photon-number state and $\left|\phi_{q}\right\rangle$ denotes the $q$ th atom's energy eigenstate, with 0 representing the ground state and 1 the excited state. As the $\left(N_{0}+1\right)$ th atom enters the cavity at time $t$, the concatenation of wave functions gives

$$
\left|\Psi_{N_{0}+1}(t)\right\rangle=\left|\Psi_{N_{0}}(t)\right\rangle \otimes\left|\Phi_{N_{0}+1}(t)\right\rangle .
$$

It is important to understand that the simple product of the two wave functions in Eq. (7) is valid only for joining two nonentangled wave functions. Once the atom is in the cavity, evolution due to atom-cavity interaction will entangle all components of the wave function so that the wave function cannot be rearranged in the form of a direct product as in Eq. (7).

The escape of an atom from the cavity requires two actions. The first is the removal of the atom-cavity interaction term pertaining to that atom from $H$. The second is the removal of contributions of that atom to the system wave function. To simplify the notation for this procedure, we assume that the last atom in the system wave function is removed. (In general, we can always relabel the atoms so that the last index is assigned to the exiting atom.) We then separate the system wave function into two groups, one of which describes the system wave function with the exiting atom in the excited state and the other describing that for the atom in the ground state. Specifically, if the atom exits at time $t^{\prime}$

$$
\begin{aligned}
& \left|\Psi_{N_{0}}\left(t^{\prime}\right)\right\rangle=\sum_{n=0}^{\infty} \underbrace{\sum_{\phi_{1}=0}^{1} \ldots \sum_{\phi_{N_{0}}=0}^{1}}_{\text {sum over } N_{0} \text { atoms }} A_{n, \phi_{1}, \phi_{2} \ldots \ldots \phi_{N_{0}}}\left(t^{\prime}\right) \\
& \times|n\rangle\left|\phi_{1}\right\rangle\left|\phi_{2}\right\rangle \cdots\left|\phi_{N_{0}}\right\rangle \\
& =\sum_{n=0}^{\infty} \underbrace{\sum_{\phi_{1}=0}^{1} \ldots \sum_{\phi_{N_{0}-1}=0}^{1}}_{\text {sum over }\left(N_{0}-1\right) \text { atoms }} A_{n, \phi_{1}, \phi_{2}, \ldots, \phi_{N_{0}-1}, 0}\left(t^{\prime}\right) \\
& \times|n\rangle\left|\phi_{1}\right\rangle\left|\phi_{2}\right\rangle \cdots\left|\phi_{N_{0}-1}\right\rangle\left|\phi_{N_{0}}=0\right\rangle \\
& +\sum_{n=0}^{\infty} \underbrace{\sum_{\phi_{1}=0}^{1} \ldots \sum_{\phi_{N_{0}-1}=0}^{1}}_{\text {sum over }\left(N_{0}-1\right) \text { atoms }} A_{n, \phi_{1}, \phi_{2} \ldots, \phi_{N_{0}-1}, 1}\left(t^{\prime}\right) \\
& \times|n\rangle\left|\phi_{1}\right\rangle\left|\phi_{2}\right\rangle \cdots\left|\phi_{N_{0}-1}\right\rangle\left|\phi_{N_{0}}=1\right\rangle \text {. }
\end{aligned}
$$

The interaction of these two groups is due to the atom-cavity interaction term for the $N_{0}$ th atom in $H_{\mathrm{JC}}$. When this atom- 
cavity interaction term goes to zero, as occurs when the atom exits the cavity, the two groups will no longer interact. This implies that from this instant onwards, while the components of the wave function in each group continue to evolve due to Rabi oscillations, there is no longer any cross entanglement between the two groups. In the simulation, when the atom exits the cavity, the probability of the atom exiting in excited (or ground) state is calculated from Eq. (8). This probability is compared with a random number between 0 and 1 . If the random number is smaller than the probability, the $N_{0}$ th atom is determined to be in the excited (or ground) state so that the wave function in Eq. (8) collapses into one group in accordance with this decision, and the ket associated with the $N_{0}$ th atom in the wave function is dropped. The result is then renormalized and the wave function continues to evolve.

In this extended QTS technique, the dissipative cavity and atom decays can be dealt with by the procedure described in Eqs. (3)-(5). The position dependence of the coupling strength can be incorporated by changing $g(\vec{r})$ over the duration of the simulation in accordance with the actual variation of coupling strength of an atom traversing the cavity. However, the QTS has its own limitations. It becomes computationally demanding as the number of atoms and photons increases. Since the atoms and photons are entangled, the number of memory locations needed to hold wave function information becomes extremely large, and at each time interval a huge amount of information needs to be processed at every coherent operation and random decay. Limitations of available workstations in memory and speed thus place a practical limit on the number of atoms which can be considered. At present, the maximum value of $N_{0}$ in our simulations is about 15 atoms [19]. The mean value of $N_{0}$, then, must be chosen so that the probability of having more than 15 atoms in the cavity is negligible. The average number of atoms in the cavity, $\langle N\rangle$, is smaller than the mean value of $N_{0}$ due to the spatial variation of the coupling constant. For example, in the fully extended simulations in Sec. III D, $\langle N\rangle \approx 3$.

\section{RESULTS}

\section{A. Thresholdlike transition by enhanced Rabi oscillation}

If $n$ photons are contained in the cavity, an initially excited atom undergoes energy exchange with the cavity (Rabi oscillation) at frequency of $2 \sqrt{n+1} g$. As the intracavity photon number increases, the rate of energy exchange will increase. For the microlaser of Ref. [4], the product of the optimal coupling constant and the atom-cavity interaction time $t_{\text {int }}$ for the most probable velocity is roughly $0.5 \mathrm{rad}$. This means that the initially excited atom will not undergo a complete Rabi oscillation if the cavity is empty. However, if the intracavity photon number is much larger than unity, the Rabi-oscillation rate will be greatly enhanced due to the factor $\sqrt{n+1}$. As the Rabi oscillation approaches a half cycle, the atom's emission probability $A_{\mathrm{em}}$ approaches unity.

To elucidate how $A_{\mathrm{em}}$ is related to the atom-cavity interaction, consider an idealized microlaser in which the atomcavity coupling constant $g$ is uniform throughout the cavity and atoms enter the cavity in an inverted state with the same velocities. If an inverted atom enters the cavity at $t=0$ and exits at $t=t_{\text {int }}$ (atom-cavity interaction time), the probability of finding the atom in the ground state at $t=t_{\text {int }}$ is simply $\sin ^{2}\left(\sqrt{n+1} g t_{\text {int }}\right)$, which is the same as $A_{\mathrm{em}}$. In steady state, the cavity field is often composed of various photon-number states, and this composition is characterized by the photonnumber distribution function $P_{n}$. The emission probability is then

$$
A_{\mathrm{em}}=\sum_{n=0}^{\infty} P_{n} \sin ^{2}\left(\sqrt{n+1} g t_{\mathrm{int}}\right) .
$$

In the experiment the atom-cavity coupling is not uniform and the atoms have different velocities, so there is no simple way to determine $A_{\mathrm{em}}$ other than realistic simulations discussed below. However, the major dependence of $A_{\mathrm{em}}$ on the atom-cavity Rabi interaction still remains.

To demonstrate the underlying principle of the microlaser thresholdlike transition, we consider the simple case of a completely inverted stream of monovelocity atoms entering the cavity and interacting with uniform coupling. Atomic decay is completely neglected. After a large number of atoms have passed through the cavity, the system evolves to an equilibrium state which can be described by a rate equation. The equation states that for the equilibrium state the number of photons decaying out of the cavity per unit time is balanced by the number of quanta emitted into the cavity by the atoms per unit time via the coherent atom-cavity interaction:

$$
\kappa\langle n\rangle=A_{\mathrm{em}}\langle N\rangle / t_{\text {int }} .
$$

We can interpret $A_{\mathrm{em}}$ as a measure of the efficiency of the system in generating photons; a large value of $A_{\mathrm{em}}$ indicates that a large proportion of energy is transferred from the atoms to the cavity.

It is possible that the simultaneous presence of two or more atoms in the cavity affects $A_{\text {em }}$ through collective interaction with a common cavity field. (See Sec. III C.) This complication can be avoided if the cavity decay rate is made so small that $\langle N\rangle$ will be much smaller than unity for the range of $\langle n\rangle$ to be studied. In this case, the chance of having two or more atoms in the cavity simultaneously can be made extremely small.

Based on these considerations, we first ran quantum trajectory simulations for a cavity with a small decay rate (equivalent to a high finesse) and a uniform coupling constant throughout the cavity (Fig. 3). In the simulations, completely inverted two-level atoms were injected into the cavity with the same velocity but with Poissonian random arrival times. The mean number of atoms in the cavity was varied from 0.01 to 0.3 . By counting the total number of atoms exiting the cavity in the ground state and comparing it with the total number of injected atoms in each simulation, $A_{\mathrm{em}}$ can be obtained. $\langle n\rangle$ is obtained by averaging the expectation value of intracavity photon number over time. The results are shown in Figs. 4(a) and 4(b). As anticipated, $A_{\text {em }}$ grows with $\langle n\rangle$. However, from Fig. 4(a) we can see that $A_{\text {em }}$ peaks at $\langle n\rangle \approx 12$. This is because at that point, enhanced Rabi oscillation causes the atoms to undergo roughly a half Rabi cycle on average as they traverse the cavity. For larger $\langle n\rangle$, the atoms begin to return to the excited state. Since $A_{\text {em }}$ can be thought of as a measure of efficiency, by which energy from the atoms is transferred to the cavity, we see that the system 

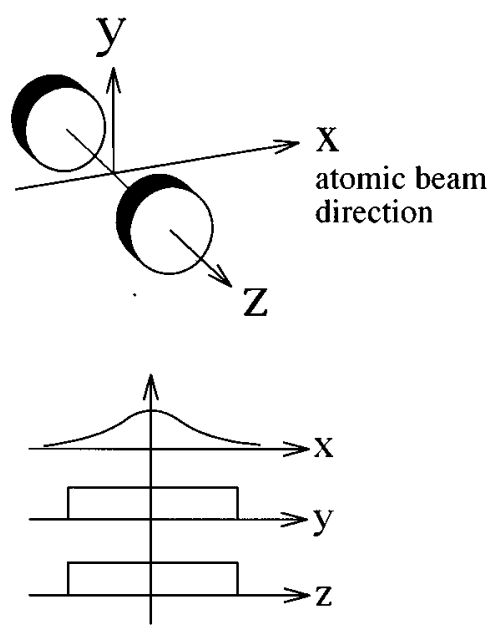

FIG. 3. Atom-cavity coupling profile of the simulation model: a Gaussian profile is assumed along the direction of atomic beam. The coupling is assumed uniform along all other directions: $g(\xi)=g_{0}$ if $|\xi|<w_{0}$, and $g(\xi)=0$ otherwise, with $\xi=x, y, z$.

is more efficient in converting the energy supplied by the atoms into laser emission at higher photon number, as long as $\langle n\rangle$ does not exceed about 12. With this interpretation, we should expect to see a nonlinear increase in $\langle n\rangle$ over a range of $\langle N\rangle$ as the system becomes more efficient, in accordance with Eq. (10). Figure 4(b) shows such a transition due to the change in efficiency.

Note that the one-atom micromaser theory of Ref. [17] can be used for this simplified model and the result can be
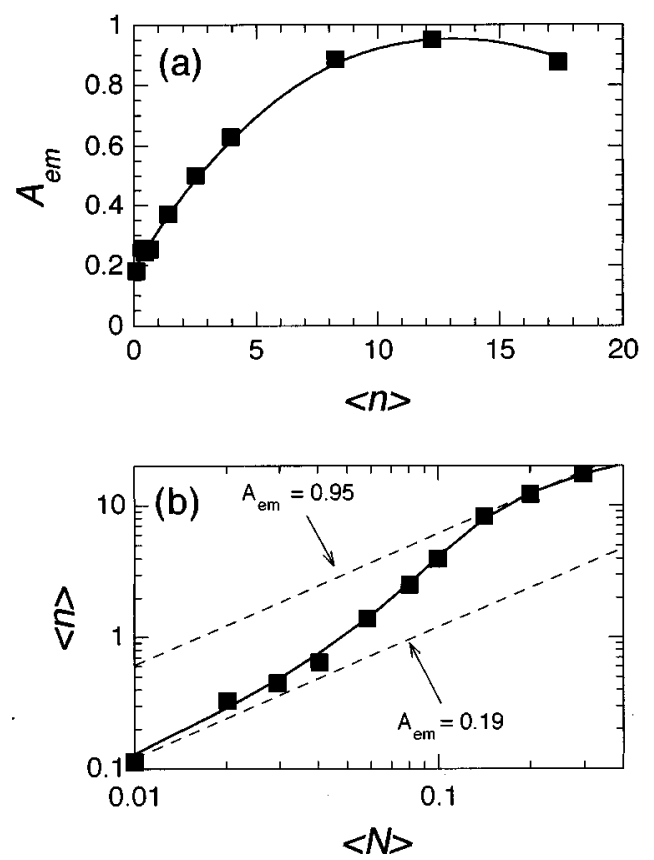

FIG. 4. Plot of (a) $A_{\text {em }}$ versus $\langle n\rangle$ and (b) $\langle n\rangle$ versus $\langle N\rangle$ from QTS simulations (boxes), assuming atom-cavity coupling profile shown in Fig. 3. The solid line shows microlaser/micromaser theoretical prediction. Coupling constant variation is shown in Fig. 3. Parameters used: $\kappa=2 \pi \times 12.5 \mathrm{kHz}, g_{0}=2 \pi \times 360 \mathrm{kHz}, v=365$ $\mathrm{m} / \mathrm{s}, w_{0}=43 \mu \mathrm{m}$. In all cases the cavity is assumed to be resonant with the atoms. compared with that of the QTS in Figs. 4(a) and 4(b). As expected, the agreement is excellent. This supports the validity of our QTS code and enables us to modify the code and apply it to more complicated configurations.

This thresholdlike transition in the microlaser can be compared to ordinary threshold behavior in the conventional laser, in which the threshold defines the transition of the laser efficiency in converting pump energy into a lasing mode. This transition is related to the laser field intensity, as governed by the conventional laser rate equations [20]:

$$
\begin{gathered}
\frac{d \bar{n}}{d t}=K(\bar{n}+1) N_{2}-K \bar{n} N_{1}-\kappa \bar{n}, \\
\frac{d N_{2}}{d t}=R_{p}-\gamma_{\mathrm{rad}} N_{2}-K \bar{n} N_{2}+K \bar{n} N_{1},
\end{gathered}
$$

$$
\frac{d N_{1}}{d t}=-\gamma_{1} N_{1}+\gamma_{\mathrm{rad}} N_{2}+K \bar{n} N_{2}-K \bar{n} N_{1}
$$

where $\bar{n}$ is the mean photon number in the cavity (equivalent to $\langle n\rangle$ in the microlaser), $R_{p}$ is a pumping rate at which the upper laser level is populated by some incoherent means, and $K=\gamma_{\mathrm{rad}} / p$ is the laser coupling coefficient, with $\gamma_{\mathrm{rad}}$ being the atomic radiative decay rate from the upper to the lower laser level and $p$ the number of cavity modes within the atomic fluorescence linewidth. $N_{1}$ and $N_{2}$ are the lower and upper laser level populations, respectively, and $\gamma_{1}$ is the atomic decay rate from the lower laser level to the ground state, with the condition that $\gamma_{1} \gg \gamma_{\text {rad }}$ in order that population inversion can occur [see Fig. 5(a)]. For conventional lasers, $p$ is very large $\left(\sim 10^{4}-10^{13}\right)$ so $K$ is much smaller than $\kappa, \gamma_{\text {rad }}$, and $\gamma_{1}$ by many orders of magnitude. In steady state, this set of equations gives

$$
\kappa \bar{n}=\left\{\frac{K\left[\gamma_{1}+\left(\gamma_{1}-\gamma_{\mathrm{rad}}+K\right) \bar{n}\right]}{\gamma_{1}\left[\gamma_{\mathrm{rad}}+K \bar{n}\right]}\right\} R_{p} .
$$

$R_{p}$ can be viewed as equivalent to $\langle N\rangle / t_{\text {int }}$ in Eq. (10); both measure the amount of energy pumped into the system per unit time. The quantity in the braces on the right hand side of the equation gives $A_{\mathrm{em}}$ in this case. It grows from $1 / p$, a very small fraction, to $1-\gamma_{\text {rad }} / \gamma_{1}$, near unity, as $\bar{n}$ increases. Plots comparable to Figs. 4(a) and 4(b) are shown in Figs. 5(b) and 5(c), respectively, for the conventional laser. Note that the sharpness of the threshold transition in the conventional laser is due to $p \gg 1$. If $p \sim 1$, the transition would be hardly distinct $[10,11]$.

However, important differences exist between the two systems despite the above-mentioned similarity. The first difference is in the transition mechanism. In the conventional laser, below threshold, emission into all available cavity modes (as many as $p$, typically $\sim 10^{4}-10^{13}$ ) takes place, so that the fraction of total emission into any particular mode is extremely small. Above threshold, coherent emission into a single lasing mode develops as stimulated emission becomes dominant. This leads to a sudden increase in the number of photons in the lasing mode. On the other hand, the microlaser has a very small $p(\sim 1)$ so that such redistribution of emission does not take place. However, the enhanced Rabioscillation process can cause more atoms to emit their pho- 

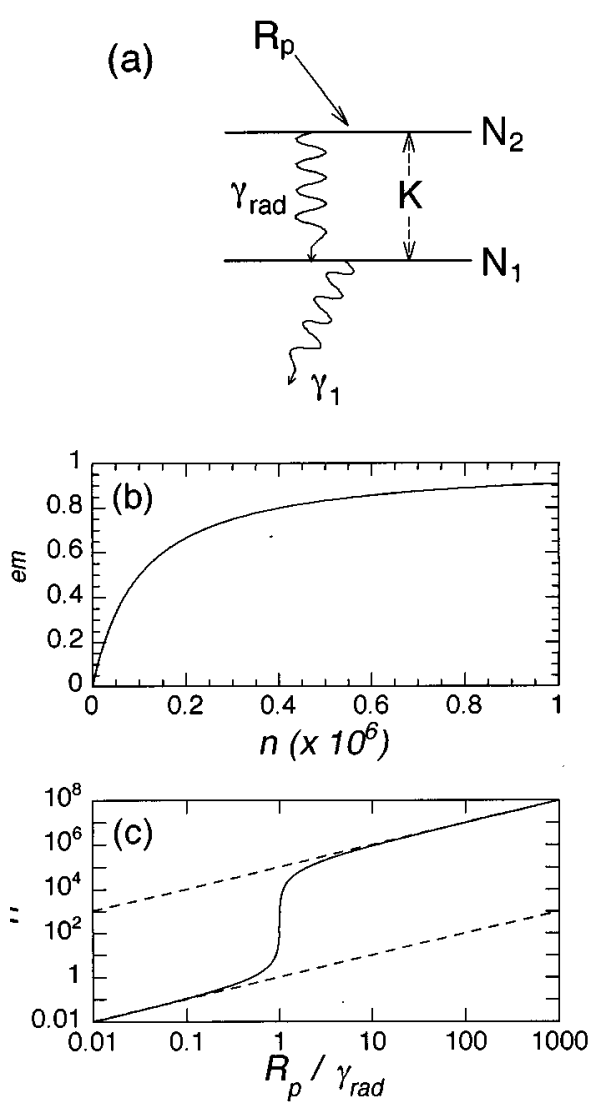

FIG. 5. (a) Energy-level diagram of the conventional laser discussed in the text. (b) Efficiency function in Eq. (14) versus $\bar{n}$, and (c) $\bar{n}$ versus $R_{p}$. Parameters used: $p=10^{5}, \gamma_{1}=2 \pi \times 20 \mathrm{MHz}$, $\kappa=2 \pi \times 2 \mathrm{MHz}, \gamma_{\mathrm{rad}}=2 \pi \times 2 \mathrm{kHz}$. These parameters were chosen to describe a typical He-Ne laser.

tons into the single mode of the cavity. When $\langle n\rangle \ll 1$ most atoms traverse the cavity without emitting photons into the cavity. As $\langle n\rangle$ grows, the enhanced Rabi oscillation enables more atoms to transfer their energy to the cavity field, hence inducing the thresholdlike transition. The second difference lies in the fact that in the microlaser $A_{\mathrm{em}}$ eventually decreases, due to the fact that further increase in $\langle n\rangle$ causes the Rabi oscillation to overshoot a half Rabi cycle. In the conventional laser, there is no equivalent process to bring about such a decrease in efficiency.

Another important characteristic of the conventional laser across threshold is the change in photon statistics, and consequently, the emission spectrum due to the transition from spontaneous emission to stimulated emission across threshold. No such drastic transition is expected in the microlaser, where photon emission into the cavity is always due to the coherent atom-cavity Rabi oscillation process. We expect that the photon statistics and, consequently, the emission spectrum of the microlaser will not dramatically differ across its thresholdlike transition. But this point is not yet well established, and needs further careful studies [21].

\section{B. Enhancement of thresholdlike transition by nonuniform coupling}

The simulations of the preceding section were based on a cavity with uniform coupling in all coordinates. The stand- (a)

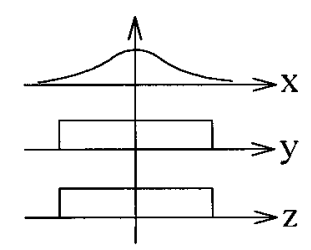

(b)

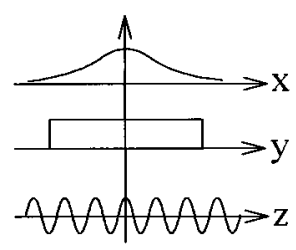

(c)

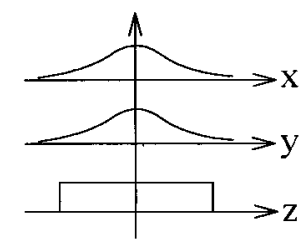

(d)

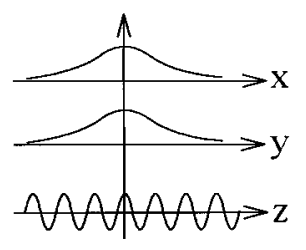

FIG. 6. Four simulation models considered in Sec. III B. They differ in position dependence of atom-cavity coupling constant. The curved single-peaked distributions represent Gaussian coupling distributions defined as $g(\xi)=g_{0} \exp \left[-\left(\xi / w_{0}\right)^{2}\right]$ with $\xi=x$ or $y$. Standing-wave distribution along $z$ direction is defined as $g(z)=g_{0} \cos [2 \pi z / \lambda]$ with $\lambda$ the wavelength. Parameters used: $w_{0}=43 \mu \mathrm{m}, \lambda=791 \mathrm{~nm}$, and $g_{0}=360 \mathrm{kHz}$.

ing-wave mode variations in coupling constant along the cavity axis and the transverse Gaussian mode profiles have been neglected to simplify the model. We next investigate the effects of these variations on $A_{\mathrm{em}}$, as well as on the $\langle n\rangle$ versus $\langle N\rangle$ curve.

The presence of a standing-wave mode in the cavity causes each atom to experience a different $g$ during its travel through the cavity. Atoms traveling close to a node experience weak coupling and undergo only weak Rabi oscillation while atoms traveling close to an antinode experience optimal coupling and undergo a maximum Rabi oscillation. We would therefore expect that only atoms in the latter case would participate significantly in the interaction with the cavity. This implies that $A_{\mathrm{em}}$ should start at a lower value than in the uniform-coupling case. However, as $\langle n\rangle$ builds up, the coupling enhancement due to more photons in the cavity will prompt even weakly coupled atoms to participate [18]. The result will be a higher ratio of maximum $A_{\mathrm{em}}$ to initial $A_{\mathrm{em}}$, thereby leading to a more distinct thresholdlike transition. The same reasoning holds for the Gaussian profile in the transverse directions.

In order to explore this effect in detail, we ran sets of simulations for the four coupling profiles depicted in Fig. 6. We summarize the resulting normalized $\langle n\rangle$ versus $\langle N\rangle$ curves in Fig. 7. As anticipated, the thresholdlike transition is more distinct for the more realistic models.

\section{Many-atom effects on thresholdlike transition}

The simulations in Secs. III A and III B were performed with a very small cavity decay rate, so that for the range of $\langle n\rangle$ values studied the probability of the cavity containing two or more atoms at any instant is very small. Note that when several atoms are present in the cavity, they can collectively interact with the common cavity field. If there are $N$ atoms with the same coupling constant $g$ within the cavity, the vacuum Rabi-oscillation frequency for these atoms as a 


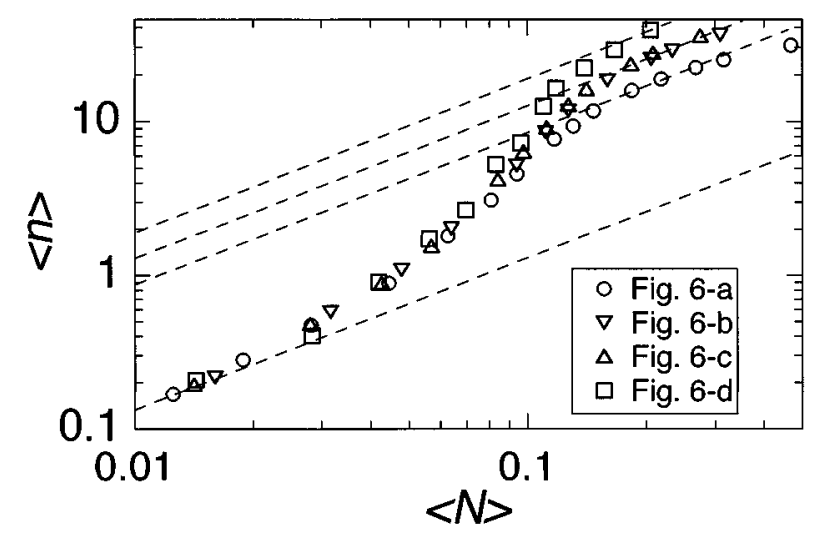

FIG. 7. Demonstration of enhancement of the thresholdlike transition by coupling constant variation.

whole is $\sqrt{N} g$ instead of $g$ [22,23]. If $N$ fluctuates in time, Rabi oscillations in the system will be complicated accordingly.

The extent of many-atom collective effects will be manifested in $A_{\mathrm{em}}$. At equilibrium, we can express $A_{\mathrm{em}}$ as a function of the time-averaged photon-number distribution function $P_{n}$ and atom-number distribution function $\mathcal{P}_{N}$. Since $\mathcal{P}_{N}$ is a Poissonian distribution, it can be completely characterized by the mean intracavity atom number $\langle N\rangle$, and hence $A_{\text {em }}$ becomes a function of $P_{n}$ and $\langle N\rangle$. Furthermore, for a given configuration of the microlaser, $P_{n}$ and $\langle n\rangle$ are expected to have a one-to-one correspondence (at least in the present operating range). Therefore $A_{\mathrm{em}}$ can be viewed as a function of $\langle n\rangle$, instead of $P_{n}$, and $\langle N\rangle$. One way of studying possible many-atom collective effects in the microlaser is then to examine the dependence of $A_{\mathrm{em}}$ on $\langle N\rangle$ for fixed $\langle n\rangle$, particularly when $\langle N\rangle \geqslant 1$.

To study this, we ran two sets of simulations. In both sets we assumed the atom-cavity coupling to be uniform as in Fig. 3 and that atoms are injected with random arrival times but with the same velocity. Atomic decay was neglected for the sake of simplicity. Hence the only randomness lies in the atom arrival times. The only difference between the two sets was that the second set employed a cavity decay rate faster than that of the first set by a factor of 19; one had a finesse of $19 \times 10^{6}$ and the other $1 \times 10^{6}$. In the set with the faster cavity decay rate, the photon storage capability of the cavity is worse, and hence, according to Eq. (10), a larger value of $\langle N\rangle$ is needed to obtain the same value of $\langle n\rangle$. For these simulations $\langle N\rangle$ was varied up to 8 , so that many-atom effects would set in.

Figure 8(a) plots both $A_{\mathrm{em}}$ curves for comparison. They turned out to be surprisingly close to each other even when $\langle N\rangle \geqslant 1$, and hence $\langle n\rangle \gg 1$ in the figure. This implies that $A_{\text {em }}$ depends almost entirely on $\langle n\rangle$ and is essentially independent of $\langle N\rangle$. There exists small difference between the two results, but since the difference is comparable to statistical error due to the stochastic nature of the simulations, we can regard both $A_{\text {em }}$ curves as practically identical. Figure 8 (b) plots the corresponding $\langle n\rangle$ versus $\langle N\rangle$ curves. To compare the shapes of the $\langle n\rangle$ versus $\langle N\rangle$ curves, the $\langle N\rangle$ value of the curve with a finesse of $19 \times 10^{6}$ was multiplied by 19 in Fig. 8(b). Again, these two curves are practically identical.
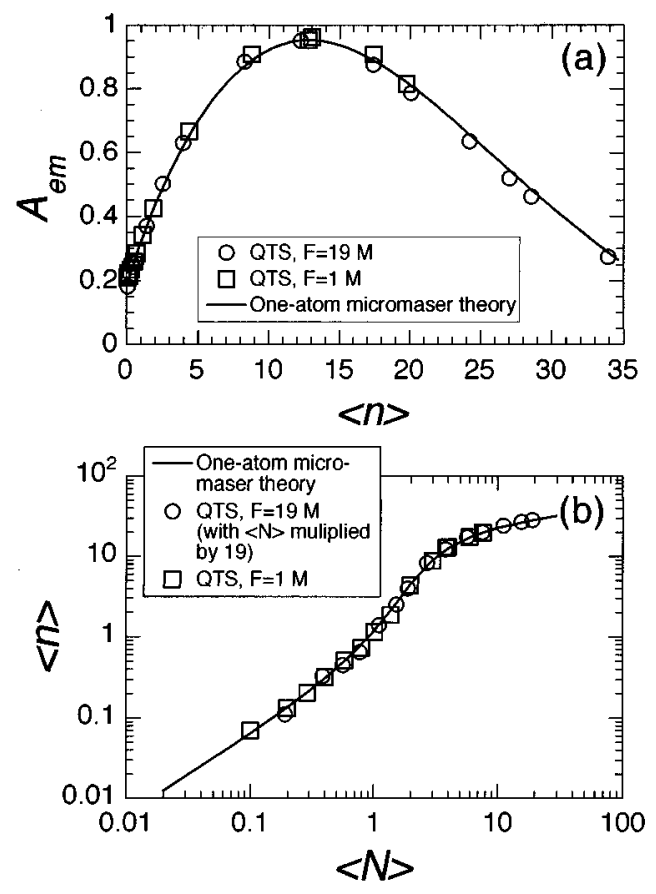

FIG. 8. Comparison of (a) emission probability $A_{\mathrm{em}}$ versus $\langle n\rangle$ and (b) $\langle n\rangle$ versus $\langle N\rangle$ curves for two different cavity decay rates. One is 19 times faster than the other. Coupling constant variation shown in Fig. 3 was used. Parameters used: $\kappa=2 \pi \times(12.5,238)$ $\mathrm{kHz} ; g_{0}=2 \pi \times 360 \mathrm{kHz}$; velocity of thermal atoms, $365 \mathrm{~m} / \mathrm{s}$; mode waist, $43 \mu \mathrm{m}$. In the legend $\mathrm{M}$ stands for $10^{6}$ (e.g., $19 \mathrm{M}$ $\left.=19 \times 10^{6}\right)$.

We thus conclude that many-atom collective effects are negligible for the idealized microlaser under consideration. Explanation of this rather surprising result lies in two facts. First, the atoms are injected at random arrival times, conforming to a Poissonian distribution, and second, the product of the coupling constant and the atom-cavity interaction time, $g t_{\text {int }}$, is much less than $\pi$. We will refer to these as the conditions of random arrival and short interaction time. Since atoms are injected into the cavity randomly in time, any coherent collective interaction of many atoms at one instant is soon interrupted by the introduction of a new atom or the departure of one of the atoms initially interacting collectively. Therefore the duration of collective effects is much shorter than the usual atom-cavity interaction time, and hence such effects would be greatly reduced. If the atomcavity interaction time is much shorter than $\pi / g$ to begin with, the many-atom collective effects would be further reduced. Conversely, if atoms are introduced into the cavity in a form of series of clusters [23], or if atoms are introduced in a regular way [24], many-atom collective effects would be significant. In addition, when a microlaser/micromaser is operating near the trapped states, i.e., $g t_{\text {int }} \sim \pi$, even occasional two-atom events severely disturb the formation of the trapped states [8].

The fact that $A_{\mathrm{em}}$ depends only on $\langle n\rangle$, independent of $\langle N\rangle$ even when $\langle N\rangle \geqslant 1$, under the condition of random arrival and short interaction time leads us to conclude that the one-atom micromaser theory can be extrapolated to the many-atom region under that condition. The solid curves in 
Figs. 8(a) and 8(b) were obtained from the micromaser theory of Ref. [17]. The curve coincides with the two simulation results, confirming our expectation.

\section{Inclusion of atomic decay and velocity distribution of injected atoms: Fitting experimental data}

Throughout the discussion we have ignored atomic decay between the two relevant states and to the $5^{3} D$ states as well. The lifetimes of these two decay processes are both about $3 \mu \mathrm{s}$. Since the atom-cavity interaction time in the experiment is about $0.2 \mu \mathrm{s}$, there is a $15 \%$ chance of atomic decay within the cavity via either of the two decay processes. QTS performed with the inclusion of these two decay processes showed little change in the overall shape of the thresholdlike transition curve. The only change observed is uniform translation of the entire curve horizontally (along the $\langle N\rangle$ axis). This is understandable when we view the added decay processes as acting to reduce the population of excited atoms, so that the intracavity atom number must be increased in order to achieve the same intracavity photon number as obtained without decay.

The other important factor regarding the realistic simulation of the experiment is the Boltzmann velocity distribution of the atoms entering the cavity. This distribution affects microlaser operation in two ways. First, atoms moving faster or slower than the most probable velocity will be underpumped or overpumped by the pump laser, respectively. In addition, the transit time of the atoms through the cavity will differ according to their respective velocities. The impact of these two differences on the thresholdlike transition is expected to be significant.

Due to constraints on the finesse of the mirrors available with current technology, the experiments of Ref. [4] were performed with a relatively large cavity decay rate compared to those of the simulations discussed so far. Therefore the thresholdlike behavior can only be seen when $\langle N\rangle$ is relatively high. In the experiment the transition occurred when $\langle N\rangle \simeq 1$.

In the simulations, the results of which are summarized in Fig. 9, we used a realistic model of the cavity which has the position-dependent coupling constant as depicted in Fig. 6(d). In addition, the atomic decay processes are included. We first performed the simulations with the actual cavity decay rate; however, due to computation limits on the ability to handle a large number of atoms in the cavity simultaneously, the simulation could only be run for the initial part of the thresholdlike transition [19].

In Sec. III C we have demonstrated that the influence of many-atom collective effects on the thresholdlike transition is negligible under the condition of random arrival and short interaction time. The microlaser experiment of Ref. [4] operates under this condition, and hence the thresholdlike transition, which occurs when $\langle N\rangle \sim 1$, should depend only on $\langle n\rangle$. Therefore we can still study the thresholdlike transition seen in the experiment by running the simulations with a very small cavity decay rate (with the other parameters unchanged), and then rescaling $\langle N\rangle$. Based on this observation, we used a cavity decay rate which is 48 times smaller than that of the actual experiment. The horizontal axis of the resulting curve was then rescaled by simply multiplying the

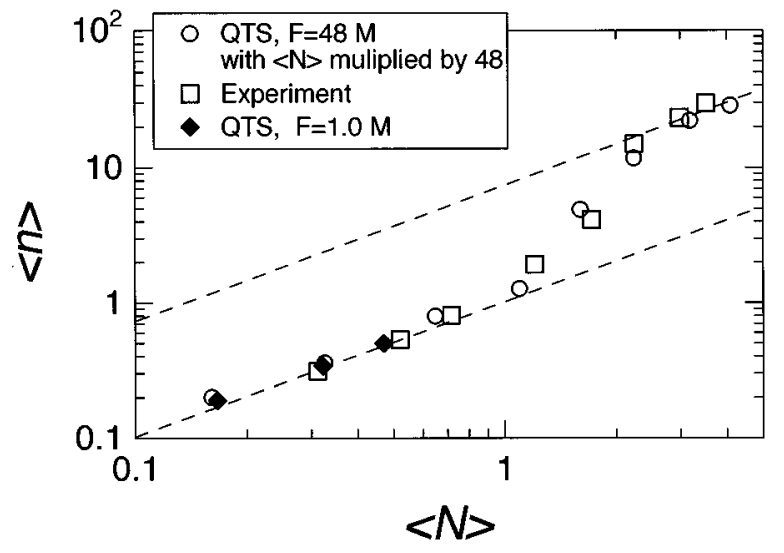

FIG. 9. Comparison of the experiment of Fig. 1 with simulation results, which include standing-wave as well as Gaussian position dependence of the coupling constant, atomic velocity distribution, and atomic spontaneous decay not only to ${ }^{1} S_{0}$ ground state but also ${ }^{3} D_{2,1}$ states. The experimental data are scaled both horizontally and vertically to match up with the simulated transition curve. The necessary scaling factors for the data are 2 and 3 for the vertical and horizontal axis, respectively. See the text for both explanation and justification of these scaling factors.

$\langle N\rangle$ values used by 48 . The results of the latter simulation agreed well with those of the former. The combined results were then regarded as the simulated transition curve for the actual experiment. The experimental data shown in Fig. 1 were then scaled both horizontally and vertically to match up with the simulated transition curve. The necessary scaling factors for the data were about 2 and 3 for the vertical and horizontal axes, respectively. These scaling factors are justified in that there exist systematic uncertainties in determining the overall atom and photon detection efficiencies in the experiment. Conversely, our simulation results can serve as a calibration method for the atom and photon detection systems. The confirmation of the goodness of fit between experiment and simulation comes from the good agreement of their respective ratio of maximum $A_{\mathrm{em}}$ to initial $A_{\mathrm{em}}$. This ratio is invariant under scaling and hence their agreement implies the validity of the simulation as well as the recalibration of detection efficiencies.

\section{CONCLUSION}

The thresholdlike transition observed in the microlaser has been studied using quantum trajectory simulations. It is found that the transition is due to the enhanced atom-cavity interaction with increase of number of intracavity photons, and the position-dependent variation of coupling constant enhances the transition. We have obtained good agreement between the full-extended simulation and the experimental data. The present work also demonstrates that many-atom collective effects in the microlaser, operating under the condition of random arrival and short interaction time (as defined in Sec. III C), are negligible. The fact that $A_{\mathrm{em}}$ depends only on mean intracavity photon number, independent of mean intracavity atom number, suggests that the one-atom 
micromaser theory of Ref. [17] can be extrapolated into the multiatom region.

Quantum trajectory simulations with many atoms in the cavity are quite demanding in computing resources. Better computing algorithms, a faster computer, and more memory would allow us to include more atoms in the simulations. For a reasonably large number of atoms, which cannot be handled by the present simulation technique, one anticipates that a simple analytic theory might be constructed. Such a theory should provide precise conditions for validity of the extrapolated one-atom theory.

\section{ACKNOWLEDGMENTS}

We thank M. S. Feld, R. R. Dasari, H.-J. Briegel, R. Glauber, and C. Fang-Yen for valuable discussions. This work is supported by the National Science Foundation under Grant No. PHY-9512056.
[1] S. Haroche and D. Kleppner, Phys. Today 42 (1), 24 (1989); S. Haroche and J. Raimond, Sci. Am. 268 (4), 54 (1993); Cavity Quantum Electrodynamics, edited by P. Berman (Academic Press, San Diego, 1994).

[2] P. Meystre et al., Opt. Lett. 79, 300 (1990); M. Brune et al., Phys. Rev. A 45, 5193 (1992); V. Buzek et al., ibid. 45, 8190 (1992); J. J. Slosser et al., Phys. Rev. Lett. 75, 418 (1995); C. Monroe et al., Science 272, 1131 (1996).

[3] Q. A. Turchette et al., Phys. Rev. Lett. 75, 4710 (1996); C. Monroe, ibid. 75, 4714 (1995), and references therein.

[4] K. An et al., Phys. Rev. Lett. 73, 3375 (1994).

[5] E. T. Jaynes and F. W. Cummings, Proc. IEEE 51, 89 (1963).

[6] D. Meschede et al., Phys. Rev. Lett. 54, 551 (1985).

[7] S. Qamar and M. S. Zubairy, Phys. Rev. A 44, 7804 (1991); M. O. Scully et al., ibid. 44, 5992 (1991); K. Vogel et al., ibid. 48, 803 (1993); K. Vogel et al., ibid. 48, 813 (1993).

[8] E. Wehner et al., Opt. Commun. 110, 655 (1994).

[9] G. Rempe et al., Phys. Rev. Lett. 67, 1727 (1991).

[10] Y. Yamamoto et al., Phys. Rev. A 44, 657 (1991).

[11] F. De Martini, F. Cairo, P. Mataloni, and F. Verzegnassi, Phys. Rev. A 46, 4220 (1992).

[12] J. Dalibard et al., Phys. Rev. Lett. 68, 580 (1991).

[13] H. J. Carmichael, Phys. Rev. Lett. 70, 2273 (1993).

[14] L. Tian and H. J. Carmichael, Phys. Rev. A 46, R6801 (1992).

[15] R. Dum et al., Phys. Rev. A 45, 4879 (1992).

[16] P. Marte et al., Phys. Rev. Lett. 71, 1335 (1993).
[17] P. Filipowicz et al., Phys. Rev. A 34, 3077 (1986).

[18] K. An and M. S. Feld, Phys. Rev. A 52, 1691 (1995).

[19] For example, the largest value of intracavity mean atom number in the realistic simulation in Sec. III D (Fig. 9) requires a total of 15 atoms to be considered in the imaginary volume $V_{0}$ in Eq. (1). If we keep up to 40 photon-number bases $[n=0,1, \ldots, 39$ in Eq. (6)], the number of coefficients to be integrated is $2^{15} \times 40 \approx 1.3 \times 10^{6}$. It takes about $10 \mathrm{~h}$ on a typical workstation to get one simulation point with reasonably good statistics.

[20] A. E. Siegman, Lasers (University Science Books, Mill Valley, CA, 1986).

[21] For the discussion of photon statistics in microcavity semiconductor lasers in the vicinity of laser threshold, see P. R. Rice and H. J. Carmichael, Phys. Rev. A 50, 4318 (1994); U. Mohideen, R. E. Slusher, P. R. Rice, and L. Pedrotti, in Quantum Electronics and Laser Science Conference, OSA Technical Digest Series Vol. 10 (Optical Society of America, Washington, D.C., 1996), p. 149.

[22] Y. Kaluzny et al., Phys. Rev. Lett. 51, 1175 (1983); G. S. Agarwal, ibid. 53, 1732 (1984); M. G. Raizen et al., ibid. 63, 240 (1989); Y. Zhu et al., ibid. 64, 2499 (1990); H.-J. Carmichael et al., Opt. Commun. 82, 73 (1991); R. J. Brecha et al., J. Opt. Soc. Am. B 12, 2329 (1995).

[23] G. M. D’Ariano et al., Phys. Rev. Lett. 74, 900 (1995).

[24] H.-J. Briegel et al., Phys. Rev. A 52, 2361 (1995). 